Document downloaded from:

http://hdl.handle.net/10251/143889

This paper must be cited as:

Ferreyra, DE.; Levis, F.; Thome, N. (15-0). Maximal classes of matrices determining generalized inverses. Applied Mathematics and Computation. 333:42-52.

https://doi.org/10.1016/j.amc.2018.03.102

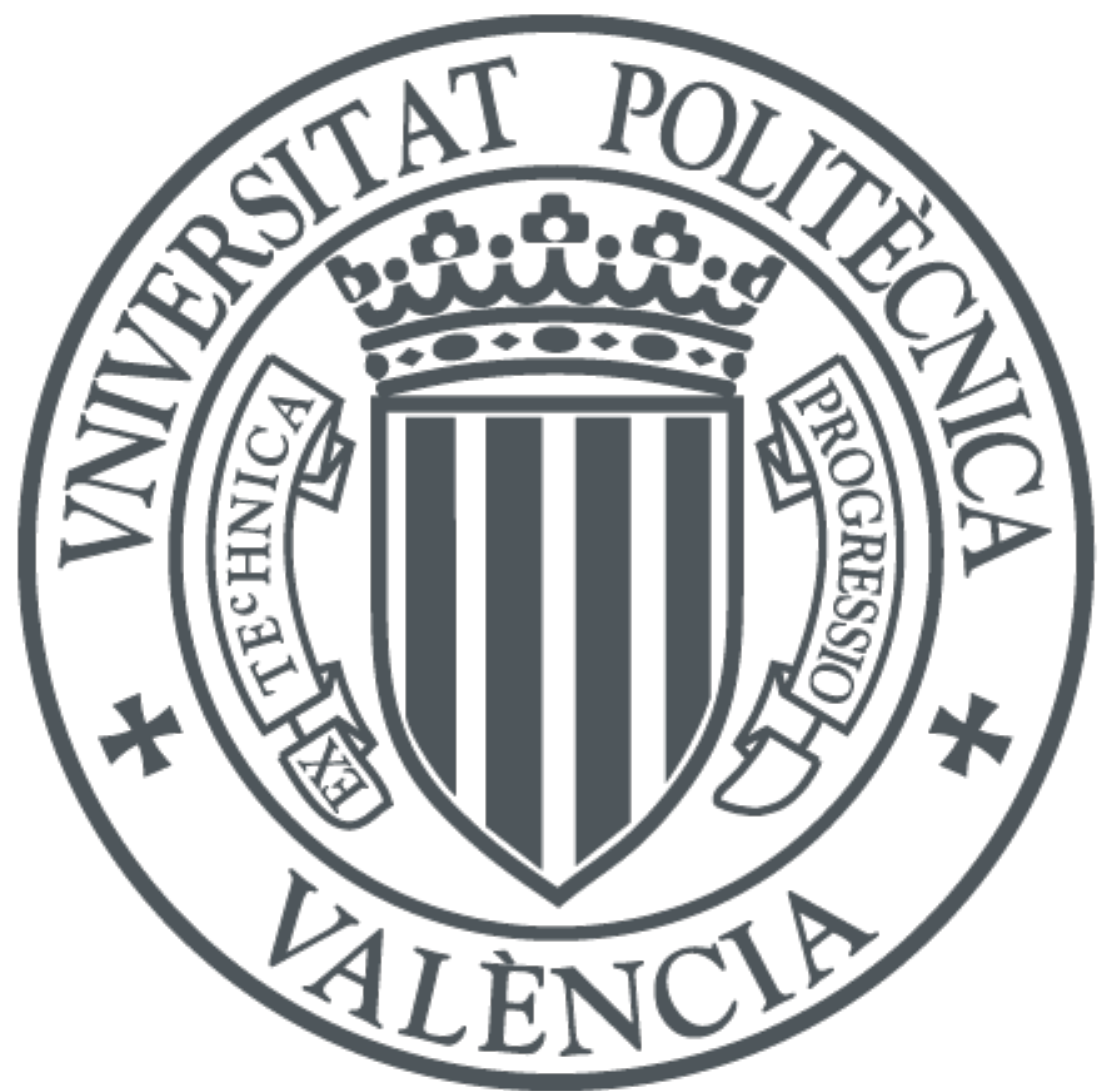

The final publication is available at

https://doi.org/10.1016/j.amc.2018.03.102

Copyright Elsevier

Additional Information 


\title{
Maximal classes of matrices determining generalized inverses
}

\author{
D.E. Ferreyra*, F.E. Levis*, N. Thome $^{\dagger}$
}

\begin{abstract}
This paper derives some further results on recent generalized inverses studied in the literature, namely core EP, DMP, and CMP inverses. Our main aim is to develop maximal classes of matrices for which their representations remain valid.
\end{abstract}

AMS Classification: 15A09

Keywords: Generalized inverses, core EP inverse, DMP inverse, CMP inverse.

\section{Introduction}

Let $\mathbb{C}^{n \times n}$ be the set of $n \times n$ complex matrices. For $A \in \mathbb{C}^{n \times n}$, the symbols $A^{*}, A^{-1}, \operatorname{rk}(A), \mathcal{N}(A)$, and $\mathcal{R}(A)$ will denote the conjugate transpose, the inverse, the rank, the kernel, and the range space of $A$, respectively. Moreover, $I_{n}$ will refer to the $n \times n$ identity matrix.

Let $A \in \mathbb{C}^{n \times n}$. We recall that the unique matrix $X \in \mathbb{C}^{n \times n}$ satisfying

$$
\text { (1) } A X A=A, \quad(2) X A X=X, \quad(3)(A X)^{*}=A X, \quad \text { and } \quad(4)(X A)^{*}=X A \text {, }
$$

is called the Moore-Penrose inverse of $A$ and is denoted by $A^{\dagger}$.

For a given complex square matrix $A$, the index of $A$, denoted by $\operatorname{Ind}(A)$, is the smallest nonnegative integer $k$ such that $\mathcal{R}\left(A^{k}\right)=\mathcal{R}\left(A^{k+1}\right)$. We observe that the index of a nonsingular matrix $A$ is 0 , and by convention, the index of the null matrix is 1 .

We also recall that the Drazin inverse of $A \in \mathbb{C}^{n \times n}$ is the unique matrix $X \in \mathbb{C}^{n \times n}$ such that

$$
\text { (2) } X A X=X, \quad \text { (5) } A X=X A, \quad \text { and } \quad\left(6^{k}\right) A^{k+1} X=A^{k}
$$

\footnotetext{
*Universidad Nacional de Río Cuarto, CONICET, FCEFQyN, RN 36 Km 601, Río Cuarto, 5800, Córdoba. Argentina.
} E-mail: deferreyra@exa.unrc.edu.ar, flevis@exa.unrc.edu.ar. Partially supported by a CONICET's Posdoctoral Research Fellowship, UNRC (grant PPI 18/C472) and CONICET (grant PIP 112-201501-00433CO).

†Instituto Universitario de Matemática Multidisciplinar, Universitat Politècnica de València, 46022, Valencia, Spain. E-mail: njthome@mat.upv.es. Partially supported by Ministerio de Economía y Competitividad of Spain grant DGI MTM2013-43678-P. 
where $k=\operatorname{Ind}(A)$, and is denoted by $A^{d}$. If $A \in \mathbb{C}^{n \times n}$ satisfies $\operatorname{Ind}(A) \leq 1$, then the Drazin inverse of $A$ is called the group inverse of $A$ and is denoted by $A^{\#}$.

A matrix $X \in \mathbb{C}^{n \times n}$ that satisfies the only equality $A X A=A$ is called an inner inverse or $\{1\}$ inverse of $A$, and a matrix $X \in \mathbb{C}^{n \times n}$ that satisfies the unique equality $X A X=X$ is called an outer inverse or $\{2\}$-inverse of $A$. In general, the set of matrices satisfying the conditions (i), $(\mathrm{j}), \ldots$ is denoted by $A\{i, j, \ldots\}$. A matrix $X \in A\{i, j, \ldots\}$ is called an $\{i, j, \ldots\}$-inverse of $A$, and is denoted by $A^{(i, j, \ldots)}$. Let $P_{A}:=A A^{\dagger}$ and $Q_{A}:=A^{\dagger} A$ denote the orthogonal projectors onto the range of $A$ and the range of $A^{*}$, respectively. Similarly, $P_{A^{\ell}}:=A^{\ell}\left(A^{\ell}\right)^{\dagger}$ and $Q_{A^{\ell}}:=\left(A^{\ell}\right)^{\dagger} A^{\ell}$ for any positive integer $\ell$.

The core inverse was introduced by Baksalary and Trenkler in [2] and later investigated by S. Malik in [7] and S.Z. Xu, J.L. Chen, X.X. Zhang in [15], among others. For a given matrix $A \in \mathbb{C}^{n \times n}$, it is defined as the unique matrix $X \in \mathbb{C}^{n \times n}$ that satisfies the conditions

$$
A X=P_{A} \quad \text { and } \quad \mathcal{R}(X) \subseteq \mathcal{R}(A)
$$

In case that such a matrix $X$ exists, it is denoted by $A^{\boxplus}$. Moreover, it was proved that $A$ is core invertible if and only if $\operatorname{Ind}(A) \leq 1$.

Two generalizations of the core inverse were introduced for $n \times n$ complex matrices, namely core EP inverses and DMP inverses. In order to recall these concepts we assume that $\operatorname{Ind}(A)=k$ for a given matrix $A \in \mathbb{C}^{n \times n}$. Firstly, the unique matrix $X \in \mathbb{C}^{n \times n}$ such that

$$
X A X=X \quad \text { and } \quad \mathcal{R}(X)=\mathcal{R}\left(X^{*}\right)=\mathcal{R}\left(A^{k}\right)
$$

is called the core EP inverse of $A$ and is denoted by $A^{\oplus}$ [11]. In [11, Lemma 3.3] K.M. Prasad and K.S. Mohana proved that the core EP of a matrix $A \in \mathbb{C}^{n \times n}$ is the unique solution of

$$
X A^{k+1}=A^{k}, \quad X A X=X, \quad(A X)^{*}=A X, \quad \text { and } \quad \mathcal{R}(X) \subseteq \mathcal{R}\left(A^{k}\right) .
$$

Recently, in [4, Theorem 2.7], it is was proved that the core EP inverse can be expressed as

$$
A^{\oplus}=\left(A P_{A^{k}}\right)^{\dagger}
$$

Secondly, the concept of DMP inverse of $A$ was introduced in [8] by S. Malik and N. Thome. In this case, the unique matrix $X \in \mathbb{C}^{n \times n}$ satisfying

$$
X A X=X, \quad X A=A^{d} A, \quad \text { and } \quad A^{k} X=A^{k} A^{\dagger},
$$

is called the DMP inverse of $A$ and is denoted by $A^{d, \dagger}$. Moreover, it was proved that

$$
A^{d, \dagger}=A^{d} A A^{\dagger}
$$


In addition, a new generalized inverse was investigated in [9] by M. Mehdipour and A. Salemi. In this case, the matrix

$$
A^{c, \dagger}=Q_{A} A^{d} P_{A},
$$

is called the CMP inverse of $A$.

The well-known Urquhart formula [1, p. 48] establishes that

$$
A^{\dagger}=A^{(1,4)} A A^{(1,3)},
$$

that is it allows us to represent the Moore-Penrose inverse by means of any $\{1,4\}$-inverse and any $\{1,3\}$-inverse of $A$. Similarly, it occurs with the Mitra [10] and Zlobec [16] formula given by

$$
A^{\dagger}=A^{*} Y A^{*},
$$

for arbitrary $Y \in\left(A^{*} A A^{*}\right)\{1\}$ and the Decell formula [3, Corollary 1] given by

$$
A^{\dagger}=A^{*} X A Y A^{*}
$$

where $X$ and $Y$ are any element in $\left(A A^{*}\right)\{1\}$ and $\left(A^{*} A\right)\{1\}$, respectively. Related to the group inverse, it is well known [1, p. 168] that

$$
A^{\#}=A X A
$$

where $X$ is an arbitrary element in $\left(A^{3}\right)\{1\}$, or more generally, for the Drazin inverse it holds

$$
A^{d}=A^{\ell} X A^{\ell}
$$

for $\ell$ being any integer not less than the index of $A$, and $X$ being an arbitrary element in $\left(A^{2 \ell+1}\right)\{1\}$.

The common fact is that all these formulas are represented by matrices in a more general class. Our main aim is to develop maximal classes for the most recent generalized inverses investigated in the literature, namely core EP, DMP, and CMP inverses.

For any matrix $A \in \mathbb{C}^{n \times n}$ of rank $r>0$, the Hartwig-Spindelböck decomposition is given by

$$
A=U\left[\begin{array}{cc}
\Sigma K & \Sigma L \\
0 & 0
\end{array}\right] U^{*},
$$

where $U \in \mathbb{C}^{n \times n}$ is unitary, $\Sigma=\operatorname{diag}\left(\sigma_{1} I_{r_{1}}, \sigma_{2} I_{r_{2}}, \ldots, \sigma_{t} I_{r_{t}}\right)$ is a diagonal matrix, the diagonal entries $\sigma_{i}$ being singular values of $A, \sigma_{1}>\sigma_{2}>\cdots>\sigma_{t}>0, r_{1}+r_{2}+\cdots+r_{t}=r$, and $K \in \mathbb{C}^{r \times r}$, $L \in \mathbb{C}^{r \times(n-r)}$ satisfy $K K^{*}+L L^{*}=I_{r}$.

On the other hand, the following two results were recently obtained by H. Kurata in [6]. 
Proposition 1.1. ([6, Theorem 3]) Let $A \in \mathbb{C}^{n \times n}$ be a matrix of index 1 written as in (1.9). Let $r=\operatorname{rk}(A)$ and $X, Y \in A\{1\}$. The following conditions are equivalent:

(a) $A^{\boxplus}=X A Y$;

(b) $\mathcal{R}(X A) \subseteq \mathcal{R}(A)$ and $Y \in A\{3\}$;

(c) $X$ and $Y$ can be expressed respectively as

$$
X=U\left[\begin{array}{cc}
(\Sigma K)^{-1} & X_{12} \\
0 & X_{22}
\end{array}\right] U^{*} \quad \text { and } \quad Y=U\left[\begin{array}{cc}
(\Sigma K)^{-1}-K^{-1} L Y_{21} & -K^{-1} L Y_{22} \\
Y_{21} & Y_{22}
\end{array}\right] U^{*}
$$

for some $X_{12} \in \mathbb{C}^{r \times(n-r)}, X_{22}, Y_{22} \in \mathbb{C}^{(n-r) \times(n-r)}$, and $Y_{21} \in \mathbb{C}^{(n-r) \times r}$.

Notice that the condition $\mathcal{R}(X A) \subseteq \mathcal{R}(A)$ in (b) is equivalent to $\mathcal{R}(X A)=\mathcal{R}(A)$ and any of them is valid for being used in (b).

Proposition 1.2. ([6, Theorem 4]) Let $A \in \mathbb{C}^{n \times n}$ be a matrix of index 1 written as in (1.9). Let $r=\operatorname{rk}(A)$ and $Z \in A\{1\}$. The following conditions are equivalent:

(a) $A^{\oplus}=\left(A^{2} Z\right)^{\dagger}$ holds;

(b) $Z \in A\{3\}$;

(c) $Z$ can be expressed as

$$
Z=U\left[\begin{array}{cc}
(\Sigma K)^{-1}-K^{-1} L Z_{21} & -K^{-1} L Z_{22} \\
Z_{21} & Z_{22}
\end{array}\right] U^{*}
$$

for some $Z_{21} \in \mathbb{C}^{(n-r) \times r}$ and $Z_{22} \in \mathbb{C}^{(n-r) \times(n-r)}$.

This paper is organized as follows. In Section 2, we discuss Proposition 1.1 and derive its extension to matrices of arbitrary index (DMP inverses) by using the Hartwig-Spindelböck decomposition (see (1.9)) besides to provide some new equivalent conditions for the core inverse. In Section 3, we give further results on two representations for core EP inverses by extending Proposition 1.1 and Proposition 1.2 to matrices of arbitrary index by using the core EP decomposition (see (3.1)). In Section 4, we use a representation of the CMP inverse obtained by Mehdipour and Salemi to derive maximal classes of matrices for which their representation remains valid. 


\section{Maximal classes for matrices determining the DMP inverse}

This section is devoted to extend Proposition 1.1 from core inverse to DMP inverse, that is, from matrices of index at most 1 to matrices of arbitrary index.

We start this section recalling an auxiliary lemma.

Lemma 2.1. ([1, p. 52]) Let $A, B, C \in \mathbb{C}^{n \times n}$. Then the matrix equation $A X B=C$ is consistent if and only if for some $A^{(1)} \in A\{1\}, B^{(1)} \in B\{1\}$,

$$
A A^{(1)} C B^{(1)} B=C,
$$

in which case the general solution is

$$
X=A^{(1)} C B^{(1)}+Z-A^{(1)} A Z B B^{(1)},
$$

for arbitrary $Z \in \mathbb{C}^{n \times n}$.

Theorem 2.2. Let $A \in \mathbb{C}^{n \times n}$ be a matrix of index $k$ written as in (1.9). Let $r=\operatorname{rk}(A), X \in A\left\{5,6^{k}\right\}$, and $Y \in A\{1\}$. The following conditions are equivalent:

(a) $A^{d, \dagger}=X A Y$;

(b) $X A=A^{d} A, A^{k} Y=A^{k} A^{\dagger}$;

(c) $X=A^{d}+Z\left(I_{n}-P_{A}\right), Y=A^{\dagger}+\left(I_{n}-Q_{A^{k}}\right) W$ for arbitrary $Z, W \in \mathbb{C}^{n \times n}$;

(d) $X$ and $Y$ can be expressed as

$$
X=U\left[\begin{array}{cc}
(\Sigma K)^{d} & Z_{12} \\
0 & Z_{22}
\end{array}\right] U^{*}
$$

and

$$
Y=U\left[\begin{array}{cc}
K^{*} \Sigma^{-1}+\left(I_{r}-K^{*} M K\right) W_{11}-K^{*} M L W_{21} & \left(I_{r}-K^{*} M K\right) W_{12}-K^{*} M L W_{22} \\
L^{*} \Sigma^{-1}-L^{*} M K W_{11}+\left(I_{n-r}-L^{*} M L\right) W_{21} & M K W_{12}+\left(I_{n-r}-L^{*} M L\right) W_{22}
\end{array}\right] U^{*}
$$

where $M:=Q_{(\Sigma K)^{k-1} \Sigma}=\left[(\Sigma K)^{k-1} \Sigma\right]^{\dagger}(\Sigma K)^{k-1} \Sigma$, for arbitrary $Z_{12}, Z_{22}, W_{11}, W_{21}, W_{12}, W_{22}$.

(e) $\mathcal{R}(X A)=\mathcal{R}\left(A^{k}\right)$ and $\mathcal{N}\left(A^{*}\right) \subseteq \mathcal{N}\left(A^{k} Y\right)$.

Proof. (a) $\Longrightarrow$ (b) Postmultiplying $A^{d, \dagger}=X A Y$ by $A$ we get $A^{d, \dagger} A=X A Y A=X A$ because $Y \in$ $A\{1\}$. Now, from $A^{d, \dagger}=A^{d} A A^{\dagger}$ we obtain

$$
X A=A^{d, \dagger} A=A^{d} A A^{\dagger} A=A^{d} A .
$$


On the other hand, premultiplying $A^{d, \dagger}=X A Y$ by $A^{k}$ we have

$$
A^{k} A^{d, \dagger}=A^{k} X A Y=A^{k+1} X Y=A^{k} Y
$$

since $X \in A\left\{5,6^{k}\right\}$. By the definition of Drazin inverse, it then follows that

$$
A^{k} Y=A^{k} A^{d, \dagger}=A^{k} A^{d} A A^{\dagger}=A^{k+1} A^{d} A^{\dagger}=A^{k} A^{\dagger} .
$$

(b) $\Longrightarrow(\mathrm{c})$ It is evident that $A^{d}$ satisfies the equation

$$
A^{d} A=X A \text {. }
$$

Since all solutions of equation (2.1) are obtained as a sum of a particular solution of (2.1) and the general solution of the homogeneous equation $X A=0$, applying Lemma 2.1 to this last equation, the general solution of (2.1) is given by

$$
X=A^{d}+Z\left(I_{n}-P_{A}\right), \quad \text { for arbitrary } Z \in \mathbb{C}^{n \times n} .
$$

Again, by Lemma 2.1, we obtain that the general solution of the equation $A^{k} A^{\dagger}=A^{k} Y$ is given by

$$
Y=A^{\dagger}+\left(I_{n}-Q_{A^{k}}\right) W, \quad \text { for arbitrary } W \in \mathbb{C}^{n \times n} .
$$

(c) $\Longrightarrow$ (a) Assume that $X=A^{d}+Z\left(I_{n}-P_{A}\right)$ and $Y=A^{\dagger}+\left(I_{n}-Q_{A^{k}}\right) W$ for arbitrary $Z, W \in \mathbb{C}^{n \times n}$. Taking into account that $A^{d} A$ is a projector and $A^{d} A=A A^{d}$, we have $A^{d} A=\left(A^{d} A\right)^{k}=\left(A^{d}\right)^{k} A^{k}$. Therefore,

$$
\begin{aligned}
X A Y & =\left[A^{d}+Z\left(I_{n}-P_{A}\right)\right] A\left[A^{\dagger}+\left(I_{n}-Q_{A^{k}}\right) W\right] \\
& =A^{d} A\left(A^{\dagger}+\left(I_{n}-Q_{A^{k}}\right) W\right) \\
& =A^{d} A A^{\dagger}+\left(A^{d}\right)^{k} A^{k}\left(I_{n}-Q_{A^{k}}\right) W \\
& =A^{d, \dagger}
\end{aligned}
$$

(c) $\Longleftrightarrow$ (d) Assume that $A$ has the form given in (1.9). From [2, Formula (1.13)],

$$
A^{\dagger}=U\left[\begin{array}{cc}
K^{*} \Sigma^{-1} & 0 \\
L^{*} \Sigma^{-1} & 0
\end{array}\right] U^{*}
$$

and then

$$
I_{n}-P_{A}=U\left[\begin{array}{cc}
0 & 0 \\
0 & I_{n-r}
\end{array}\right] U^{*} .
$$


From [8, Formula (14)] we have

$$
A^{d}=U\left[\begin{array}{cc}
(\Sigma K)^{d} & {\left[(\Sigma K)^{d}\right]^{2} \Sigma L} \\
0 & 0
\end{array}\right] U^{*}
$$

Next, partitioning accordingly

$$
Z=U\left[\begin{array}{ll}
Z_{11} & \widetilde{Z}_{12} \\
Z_{21} & Z_{22}
\end{array}\right] U^{*}
$$

a straightforward computation shows that $X=A^{d}+Z\left(I_{n}-P_{A}\right)$ is equivalent to

$$
X=U\left[\begin{array}{cc}
(\Sigma K)^{d} & Z_{12} \\
0 & Z_{22}
\end{array}\right] U^{*}
$$

for arbitrary $Z_{12}$ and $Z_{22}$, after renaming adequately a block.

On the other hand, since

$$
A^{k}=U\left[\begin{array}{cc}
(\Sigma K)^{k} & (\Sigma K)^{k-1} \Sigma L \\
0 & 0
\end{array}\right] U^{*}
$$

from [5, Lemma 1] we have

$$
\begin{aligned}
\left(A^{k}\right)^{\dagger} & =U\left[\begin{array}{cc}
\Phi_{1} & \Phi_{2} \\
0 & 0
\end{array}\right]^{\dagger} U^{*}=U\left(\left[\begin{array}{cc}
0 & I_{r} \\
I_{n-r} & 0
\end{array}\right]\left[\begin{array}{cc}
0 & 0 \\
\Phi_{2} & \Phi_{1}
\end{array}\right]\left[\begin{array}{cc}
0 & I_{n-r} \\
I_{r} & 0
\end{array}\right]\right)^{\dagger} U^{*} \\
& =U\left[\begin{array}{cc}
0 & I_{n-r} \\
I_{r} & 0
\end{array}\right]\left[\begin{array}{cc}
0 & 0 \\
\Phi_{2} & \Phi_{1}
\end{array}\right]^{\dagger}\left[\begin{array}{cc}
0 & I_{r} \\
I_{n-r} & 0
\end{array}\right] U^{*}=U\left[\begin{array}{cc}
\Phi_{1}^{*} R^{\dagger} & 0 \\
\Phi_{2}^{*} R^{\dagger} & 0
\end{array}\right] U^{*},
\end{aligned}
$$

where $R=\Phi_{1} \Phi_{1}^{*}+\Phi_{2} \Phi_{2}^{*}, \Phi_{1}=(\Sigma K)^{k}$, and $\Phi_{2}=(\Sigma K)^{k-1} \Sigma L$. Hence,

$$
Q_{A^{k}}=\left(A^{k}\right)^{\dagger} A^{k}=U\left[\begin{array}{cc}
\Phi_{1}^{*} R^{\dagger} \Phi_{1} & \Phi_{1}^{*} R^{\dagger} \Phi_{2} \\
\Phi_{2}^{*} R^{\dagger} \Phi_{1} & \Phi_{2}^{*} R^{\dagger} \Phi_{2}
\end{array}\right] U^{*},
$$

Now, we calculate $R$ as follows

$$
\begin{aligned}
R & =\left[\begin{array}{ll}
\Phi_{1} & \Phi_{2}
\end{array}\right]\left[\begin{array}{ll}
\Phi_{1} & \Phi_{2}
\end{array}\right]^{*} \\
& =\left[\begin{array}{ll}
(\Sigma K)^{k} & (\Sigma K)^{k-1} \Sigma L
\end{array}\right]\left[\begin{array}{l}
\left.K^{*}(\Sigma K)^{k-1} \Sigma\right)^{*} \\
L^{*}\left((\Sigma K)^{k-1} \Sigma\right)^{*}
\end{array}\right] \\
& =(\Sigma K)^{k-1} \Sigma\left[\begin{array}{ll}
K & L
\end{array}\right]\left[\begin{array}{c}
K^{*} \\
L^{*}
\end{array}\right]\left((\Sigma K)^{k-1} \Sigma\right)^{*} \\
& =(\Sigma K)^{k-1} \Sigma\left((\Sigma K)^{k-1} \Sigma\right)^{*} .
\end{aligned}
$$


We know that $B^{\dagger}=B^{*}\left(B B^{*}\right)^{\dagger}$ for any complex matrix $B$. In consequence, after computing $\Phi_{1}^{*} R^{\dagger}$ and $\Phi_{2}^{*} R^{\dagger}$ we get

$$
I_{n}-Q_{A^{k}}=U\left[\begin{array}{cc}
I_{r}-K^{*}\left[(\Sigma K)^{k-1} \Sigma\right]^{\dagger}(\Sigma K)^{k} & -K^{*}\left[(\Sigma K)^{k-1} \Sigma\right]^{\dagger}(\Sigma K)^{k-1} \Sigma L \\
-L^{*}\left[(\Sigma K)^{k-1} \Sigma\right]^{\dagger}(\Sigma K)^{k} & I_{n-r}-L^{*}\left[(\Sigma K)^{k-1} \Sigma\right]^{\dagger}(\Sigma K)^{k-1} \Sigma L
\end{array}\right] U^{*}
$$

Now, partitioning adequately

$$
W=U\left[\begin{array}{ll}
W_{11} & W_{12} \\
W_{21} & W_{22}
\end{array}\right] U^{*}
$$

from (2.2) it is clear that the equalities $Y=A^{\dagger}+\left(I_{n}-Q_{A^{k}}\right) W$ is equivalent to the expression for $Y$ given in item $(d)$.

(b) $\Longrightarrow$ (e) Clearly, $X A=A^{d} A$ yields $\mathcal{R}(X A)=\mathcal{R}\left(A^{d} A\right)=\mathcal{R}\left(A^{k}\right)$. On the other hand, from $A^{k} Y=A^{k} A^{\dagger}$ we obtain $\mathcal{N}\left(A^{*}\right)=\mathcal{N}\left(A^{\dagger}\right) \subseteq \mathcal{N}\left(A^{k} A^{\dagger}\right)=\mathcal{N}\left(A^{k} Y\right)$.

(e) $\Longrightarrow$ (b) Since the matrix $A$ is written as in (1.9), we can consider a partition accordingly

$$
X=U\left[\begin{array}{ll}
X_{11} & X_{12} \\
X_{21} & X_{22}
\end{array}\right] U^{*}
$$

From $A X=X A$ we obtain

$$
\begin{gathered}
X_{11} \Sigma K=\Sigma K X_{11}+\Sigma L X_{21}, \\
X_{21} \Sigma K=0, \\
X_{21} \Sigma L=0 .
\end{gathered}
$$

Postmultiplying (2.5) by $K^{*},(2.6)$ by $L^{*}$, and adding them we obtain $X_{21} \Sigma\left(K K^{*}+L L^{*}\right)=0$. Thus, $X_{21}=0$. Therefore, from (2.4) we get

$$
X_{11}(\Sigma K)=(\Sigma K) X_{11}
$$

Moreover, the equality $A^{k+1} X=A^{k}$ implies $(\Sigma K)^{k+1} X_{11}=(\Sigma K)^{k}$ and then

$$
(\Sigma K)^{k} X_{11}=(\Sigma K)^{k-1}
$$

because of [8, Lemma 2.8] guarantees that ind $(A)=k$ implies ind $(\Sigma K)=k-1$.

On the other hand, we observe that $\mathcal{R}(X A) \subseteq \mathcal{R}\left(A^{k}\right)$ can be equivalently expressed as $P_{A^{k}} X A=X A$. By [4, Theorem 3.2] we get

$$
P_{A^{k}}=A^{k}\left(A^{k}\right)^{\dagger}=U\left[\begin{array}{cc}
(\Sigma K)^{k-1}\left((\Sigma K)^{k-1}\right)^{\dagger} & 0 \\
0 & 0
\end{array}\right] U^{*}=U\left[\begin{array}{cc}
P_{(\Sigma K)^{k-1}} & 0 \\
0 & 0
\end{array}\right] U^{*} .
$$


Since

$$
X A=U\left[\begin{array}{ll}
X_{11} \Sigma K & X_{11} \Sigma L \\
X_{21} \Sigma K & X_{21} \Sigma L
\end{array}\right] U^{*}=U\left[\begin{array}{cc}
X_{11} \Sigma K & X_{11} \Sigma L \\
0 & 0
\end{array}\right] U^{*},
$$

the condition $P_{A^{k}} X A=X A$ implies $P_{(\Sigma K)^{k-1}} X_{11} \Sigma K=X_{11} \Sigma K$ and $P_{(\Sigma K)^{k-1}} X_{11} \Sigma L=X_{11} \Sigma L$. Postmultiplying the first equation by $K^{*}$, the second by $L^{*}$, and adding them we obtain

$$
P_{(\Sigma K)^{k-1}} X_{11}=X_{11}
$$

Now, from (2.7), (2.8), and (2.10) we have

$$
X_{11}(\Sigma K) X_{11}=X_{11}(\Sigma K) P_{(\Sigma K)^{k-1}} X_{11}=\left[X_{11}(\Sigma K)^{k}\right]\left[(\Sigma K)^{k-1}\right]^{\dagger} X_{11}=P_{(\Sigma K)^{k-1}} X_{11}=X_{11} .
$$

Taking into account the fact $\operatorname{ind}(\Sigma K)=k-1$, from (2.7), (2.8), and (2.11) we get $X_{11}=(\Sigma K)^{d}$. Finally, the equality $X A=A^{d} A$ follows from an easy computation.

On the other hand, we write $Y$ as

$$
Y=U\left[\begin{array}{ll}
Y_{11} & Y_{12} \\
Y_{21} & Y_{22}
\end{array}\right] U^{*}
$$

according to the partition of $A$. Clearly, vectors of the form

$$
v=U\left[\begin{array}{l}
0 \\
y
\end{array}\right] \in \mathbb{C}^{n} \quad \text { with arbitrary } \quad y \in \mathbb{C}^{n-r},
$$

belong to $\mathcal{N}\left(A^{*}\right)$. By (e), $v \in \mathcal{N}\left(A^{k} Y\right)$ holds. In consequence, from

$$
A^{k} Y=U\left[\begin{array}{cc}
(\Sigma K)^{k} Y_{11}+(\Sigma K)^{k-1} \Sigma L Y_{21} & (\Sigma K)^{k} Y_{12}+(\Sigma K)^{k-1} \Sigma L Y_{22} \\
0 & 0
\end{array}\right] U^{*}
$$

the condition $v \in \mathcal{N}\left(A^{k} Y\right)$ implies that $\left((\Sigma K)^{k} Y_{12}+(\Sigma K)^{k-1} \Sigma L Y_{22}\right) y=0$ is valid for arbitrary $y \in \mathbb{C}^{n-r}$. Thus, $(\Sigma K)^{k} Y_{12}+(\Sigma K)^{k-1} \Sigma L Y_{22}=0$. Also, the condition $A Y A=A$ holds if and only if $\Sigma K Y_{11}+\Sigma L Y_{21}=I_{r}$, which implies that $(\Sigma K)^{k} Y_{11}+(\Sigma K)^{k-1} \Sigma L Y_{21}=(\Sigma K)^{k-1}$ is also true. Now, it is easy to check that the equality $A^{k} Y=A^{k} A^{\dagger}$ holds.

We would like to highlight the crucial fact obtained in Theorem 2.2 (b) where two decoupled equations are obtained and then the problem can be solved independently in $X$ and $Y$.

Remark 2.3. Setting $Z=W=0$ in Theorem 2.2 (c) we get $A^{d, \dagger}=A^{d} A A^{\dagger}$, which is a well-known representation for the DMP inverse of $A[8]$. 
Remark 2.4. The necessity of the general hypothesis in Theorem 2.2 arises from the idea of giving a more general expression for $A^{d, \dagger}$ (instead of $A^{d, \dagger}=A^{d} A A^{\dagger}$ ), by changing $A^{d}$ and $A^{\dagger}$ with matrices $X$ and $Y$ respectively, satisfying less restrictive properties than those satisfied by $A^{d}$ and $A^{\dagger}$. In order to do that, and following the same idea as in Proposition 1.1 where the author change $A^{\#}$ with $X \in A\{1\}$ and $A^{\dagger}$ with $Y \in A\{1\}$, we establish $X \in A\left\{5,6^{k}\right\}$ instead of $A^{d}$ and $Y \in A\{1\}$ instead of $A^{\dagger}$. We notice that both restrictions on $X$ and $Y$ are required in the proof.

Remark 2.5. The following conditions:

(c') $X=A^{d}+Z\left(I_{n}-P_{A}\right), Y=A^{\dagger}+\left(I_{n}-Q_{A^{k}}\right) W$ for some $Z, W \in \mathbb{C}^{n \times n}$;

(d') $X$ and $Y$ are as in Theorem 2.2 (c) for some $Z_{12}, Z_{22}, W_{11}, W_{21}, W_{12}$, and $W_{22}$;

are respectively equivalent to conditions (c) and (d) in Theorem 2.2. In fact, (c) $\rightarrow\left(c^{\prime}\right)$ is trivial and $\left(c^{\prime}\right) \rightarrow$ (a) follows exactly as (c) $\rightarrow$ (a) in the proof of the theorem. A similar argument yields the equivalence between $(\mathrm{d})$ and $\left(\mathrm{d}^{\prime}\right)$.

Before having stated our previous Theorem, a natural question would have been the following: For a given matrix $A \in \mathbb{C}^{n \times n}$, is it possible to introduce an outer inverse of $A$ given by $A^{d,-}:=A^{d} A A^{-}$, for some fixed $A^{-} \in A\{1\}$, having interesting properties? The answer would have been affirmative. However, this possibility is now included in the maximal class that we have found in Theorem 2.2 and it has no sense to do a study separately for this class of matrices.

\section{Maximal classes for matrices determining the Core EP in- verse}

In this section we give further results on two representations for core EP inverses extending Propositions 1.1 and 1.2 to matrices of arbitrary index by using the core EP decomposition.

According to Theorem 2.2 in [13], every matrix $A \in \mathbb{C}^{n \times n}$ with $\operatorname{Ind}(A)=k$ can be represented as

$$
A=A_{1}+A_{2}, \quad A_{1}:=U\left[\begin{array}{cc}
T & S \\
0 & 0
\end{array}\right] U^{*}, \quad A_{2}:=U\left[\begin{array}{cc}
0 & 0 \\
0 & N
\end{array}\right] U^{*},
$$

where $T$ is nonsingular with $\rho=\operatorname{rk}(T)=\operatorname{rk}\left(A^{k}\right), N$ is nilpotent of index $k$, and $U$ is unitary. The representation of $A$ given in (3.1) satisfies $\operatorname{Ind}\left(A_{1}\right) \leq 1, A_{2}^{k}=0$, and $A_{1}^{*} A_{2}=A_{2} A_{1}=0$ [13, Theorem 2.1]. Moreover, it is unique in that sense [13, Theorem 2.4] and is called the core EP decomposition of $A$. For further references we refer the reader to [14] and the references therein. 
Wang [13, Theorem 13] also gave a representation for the core EP inverse as

$$
A^{\oplus}=U\left[\begin{array}{cc}
T^{-1} & 0 \\
0 & 0
\end{array}\right] U^{*} \text {. }
$$

Furthermore, when $k=1$, the factorization (1.9) provides the core EP decomposition of $A$, where $T=\Sigma K$ and $S=\Sigma L$.

The uniqueness of the Drazin inverse allows us to show the following representation by a simple computation.

Theorem 3.1. Let $A \in \mathbb{C}^{n \times n}$ be a matrix of index $k$ written as in (3.1). Then

$$
A^{d}=U\left[\begin{array}{cc}
T^{-1} & \left(T^{k+1}\right)^{-1} \widetilde{T} \\
0 & 0
\end{array}\right] U^{*}, \quad \text { where } \quad \widetilde{T}=\sum_{j=1}^{k} T^{j} S N^{k-j} .
$$

By using (3.3), it can be also seen that the core EP inverse of a square matrix $A$ with $\operatorname{Ind}(A)=k$ can be written in the form

$$
A^{\oplus}=A^{d} P_{A^{k}} .
$$

Next, we establish the maximal classes providing the most general form to represent a DMP inverse.

Theorem 3.2. Let $A \in \mathbb{C}^{n \times n}$ be a matrix of index $k$ written as in (3.1). Let $\rho=\operatorname{rk}\left(A^{k}\right), X \in A\left\{5,6^{k}\right\}$, and $Y \in A^{k}\{1\}$. The following conditions are equivalent:

(a) $A^{\oplus}=X A^{k} Y$;

(b) $X A^{k}=A^{d} A^{k}, Y \in A^{k}\{3\}$ (or equivalently, $X A^{k}=A^{d} A^{k}, A^{k} Y=P_{A^{k}}$ );

(c) $X=A^{d}+Z\left(I_{n}-P_{A^{k}}\right), \quad Y=\left(A^{k}\right)^{\dagger}+\left(I_{n}-Q_{A^{k}}\right) W$ for arbitrary $Z, W \in \mathbb{C}^{n \times n}$;

(d) $X$ and $Y$ can be expressed as

$$
X=U\left[\begin{array}{cc}
T^{-1} & X_{12} \\
0 & X_{22}
\end{array}\right] U^{*}, \quad Y=U\left[\begin{array}{cc}
\left(T^{k}\right)^{-1}\left(I_{\rho}-\widetilde{T} Y_{21}\right) & -\left(T^{k}\right)^{-1} \widetilde{T} Y_{22} \\
Y_{21} & Y_{22}
\end{array}\right] U^{*}
$$

with $\widetilde{T}$ defined in (3.3) and for arbitrary $X_{12} \in \mathbb{C}^{r \times(n-\rho)}, X_{22}, Y_{22} \in \mathbb{C}^{(n-\rho) \times(n-\rho)}$, and $Y_{21} \in$ $\mathbb{C}^{(n-\rho) \times \rho}$.

(e) $\mathcal{R}\left(X A^{k}\right)=\mathcal{R}\left(A^{k}\right)$ and $\mathcal{N}\left(\left(A^{k}\right)^{*}\right) \subseteq \mathcal{N}\left(A^{k} Y\right)$.

Proof. (a) $\Longrightarrow$ (b) Postmultiplying $A^{\oplus}=X A^{k} Y$ by $A^{k}$ we obtain $A^{\oplus} A^{k}=X A^{k} Y A^{k}=X A^{k}$ because $Y \in A^{k}\{1\}$. So, from (3.4) we get

$$
X A^{k}=A^{\oplus} A^{k}=A^{d} P_{A^{k}} A^{k}=A^{d} A^{k} .
$$


On the other hand, by using the fact that $A A^{\oplus}=P_{A^{k}}\left[4\right.$, Lemma 2.6] and premultiplying $A^{\oplus}=X A^{k} Y$ by $A$ we have

$$
P_{A^{k}}=A A^{\oplus}=A X A^{k} Y=A^{k+1} X Y=A^{k} Y
$$

because $X \in A\left\{5,6^{k}\right\}$. The equivalence between $Y \in A^{k}\{3\}$ and $A^{k} Y=P_{A^{k}}$ is easy to see. In fact, from $A^{k} Y A^{k}=A^{k}$ we obtain that $A^{k} Y$ is a projector onto $\mathcal{R}\left(A^{k}\right)$. From $\left(A^{k} Y\right)^{*}=A^{k} Y$ we get that $A^{k} Y$ is an orthogonal projector onto $\mathcal{R}\left(A^{k}\right)$. The uniqueness of such a kind projectors leads to $A^{k} Y=A^{k}\left(A^{k}\right)^{\dagger}=P_{A^{k}}$. The converse is trivial.

(b) $\Longrightarrow$ (c) Similar arguments to that of the proof of Theorem 2.2, show that all solutions of equation $X A^{k}=A^{d} A^{k}$ are given by

$$
X=A^{d}+Z\left(I_{n}-P_{A^{k}}\right), \quad \text { for arbitrary } Z \in \mathbb{C}^{n \times n} .
$$

Analogously, the general solution of the equation $A^{k} Y=P_{A^{k}}$ is given by

$$
Y=\left(A^{k}\right)^{\dagger}+\left(I_{n}-Q_{A^{k}}\right) W, \quad \text { for arbitrary } W \in \mathbb{C}^{n \times n} .
$$

(c) $\Longrightarrow$ (a) We observe that $P_{A^{k}} A^{k}=A^{k}$ and $A^{k} Q_{A^{k}}=A^{k}$. So,

$$
\begin{aligned}
X A^{k} Y & =\left[A^{d}+Z\left(I_{n}-P_{A^{k}}\right)\right] A^{k}\left[\left(A^{k}\right)^{\dagger}+\left(I_{n}-Q_{A^{k}}\right) W\right]=A^{d} A^{k}\left[\left(A^{k}\right)^{\dagger}+\left(I_{n}-Q_{A^{k}}\right) W\right] \\
& =A^{d} A^{k}\left(A^{k}\right)^{\dagger}=A^{d} P_{A^{k}}=A^{\oplus},
\end{aligned}
$$

where the last equality follows from (3.4).

(a) $\Longrightarrow($ d) Since $A$ is written as in (3.1), we partition

$$
Y=U\left[\begin{array}{ll}
Y_{11} & Y_{12} \\
Y_{21} & Y_{22}
\end{array}\right] U^{*},
$$

according to the sizes of the partition of $A$. Since (a) $\Longrightarrow(\mathrm{c})$ is valid, we have that

$$
X=A^{d}+Z\left(I_{n}-P_{A^{k}}\right) \quad \text { for arbitrary } Z \in \mathbb{C}^{n} .
$$

From [4, Lemma 2.5]

$$
P_{A^{k}}=U\left[\begin{array}{cc}
I_{\rho} & 0 \\
0 & 0
\end{array}\right] U^{*}
$$

Now, by making the following partition with blocks of adequate sizes

$$
Z=U\left[\begin{array}{ll}
Z_{11} & Z_{12} \\
Z_{21} & Z_{22}
\end{array}\right] U^{*},
$$


from (3.3) and (3.5) we have

$$
\begin{aligned}
U^{*} X U & =\left[\begin{array}{cc}
T^{-1} & \left(T^{k+1}\right)^{-1} \widetilde{T} \\
0 & 0
\end{array}\right]+\left[\begin{array}{cc}
Z_{11} & Z_{12} \\
Z_{21} & Z_{22}
\end{array}\right]\left[\begin{array}{cc}
0 & 0 \\
0 & I_{n-\rho}
\end{array}\right] \\
& =\left[\begin{array}{cc}
T^{-1} & \left(T^{k+1}\right)^{-1} \widetilde{T}+Z_{12} \\
0 & Z_{22}
\end{array}\right] .
\end{aligned}
$$

Since $Z_{12}$ and $Z_{22}$ are arbitrary, we have that

$$
X=U\left[\begin{array}{cc}
T^{-1} & X_{12} \\
0 & X_{22}
\end{array}\right] U^{*},
$$

for arbitrary $X_{12}$ and $X_{22}$.

On the other hand, since $(\mathrm{a}) \Longrightarrow(\mathrm{b})$ has been proved, we can deduce that $\left(A^{k} Y\right)^{*}=A^{k} Y$ holds. In consequence, by comparing their blocks we arrive at $T^{k} Y_{12}+\widetilde{T} Y_{22}=0$. It then follows that $Y_{12}=-\left(T^{k}\right)^{-1} \widetilde{T} Y_{22}$. Next, since

$$
\begin{aligned}
A^{k} Y A^{k} & =U\left[\begin{array}{cc}
T^{k} & \widetilde{T} \\
0 & 0
\end{array}\right]\left[\begin{array}{cc}
Y_{11} & Y_{12} \\
Y_{21} & Y_{22}
\end{array}\right]\left[\begin{array}{cc}
T^{k} & \widetilde{T} \\
0 & 0
\end{array}\right] U^{*} \\
& =U\left[\begin{array}{cc}
\left(T^{k} Y_{11}+\widetilde{T} Y_{21}\right) T^{k} & \left(T^{k} Y_{11}+\widetilde{T} Y_{21}\right) \widetilde{T} \\
0 & 0
\end{array}\right] U^{*},
\end{aligned}
$$

the condition $A^{k} Y A^{k}=A^{k}$ implies that $T^{k} Y_{11}+\widetilde{T} Y_{21}=I_{\rho}$, from where $Y_{11}=\left(T^{k}\right)^{-1}\left(I_{\rho}-\widetilde{T} Y_{21}\right)$. Thus, (e) is derived.

(d) $\Longrightarrow$ (a) Let $\widetilde{T}$ be defined as in (3.3). An easy computation shows that

$$
\begin{aligned}
X A^{k} Y & =U\left[\begin{array}{cc}
T^{-1} & X_{12} \\
0 & X_{22}
\end{array}\right]\left[\begin{array}{cc}
T^{k} & \widetilde{T} \\
0 & 0
\end{array}\right]\left[\begin{array}{cc}
\left(T^{k}\right)^{-1}\left(I_{\rho}-\widetilde{T} Y_{21}\right) & -\left(T^{k}\right)^{-1} \widetilde{T} Y_{22} \\
Y_{21} & Y_{22}
\end{array}\right] U^{*} \\
& =U\left[\begin{array}{cc}
T^{-1} & 0 \\
0 & 0
\end{array}\right] U^{*} \\
& =A^{\oplus} .
\end{aligned}
$$

(b) $\Longrightarrow$ (e) Clearly, $X A^{k}=A^{d} A^{k}$ yields $\mathcal{R}\left(X A^{k}\right)=\mathcal{R}\left(A^{d} A^{k}\right)=\mathcal{R}\left(A^{k}\right)$. On the other hand, from $\left(A^{k} Y\right)^{*}=A^{k} Y$ we obtain $\mathcal{N}\left(\left(A^{k}\right)^{*}\right) \subseteq \mathcal{N}\left(Y^{*}\left(A^{k}\right)^{*}\right)=\mathcal{N}\left(\left(A^{k} Y\right)^{*}\right)=\mathcal{N}\left(A^{k} Y\right)$.

(e) $\Longrightarrow$ (d) Since the matrix $A$ is written as in (3.1), we can consider a partition accordingly

$$
X=U\left[\begin{array}{ll}
X_{11} & X_{12} \\
X_{21} & X_{22}
\end{array}\right] U^{*}
$$


As

$$
A^{k+1}=U\left[\begin{array}{cc}
T^{k+1} & T \widetilde{T} \\
0 & 0
\end{array}\right] U^{*},
$$

from $A^{k+1} X=A^{k}$ we obtain $X_{11}=T^{-1}$.

We observe that $\mathcal{R}\left(X A^{k}\right) \subseteq \mathcal{R}\left(A^{k}\right)$ can be equivalently expressed as $P_{A^{k}} X A^{k}=X A^{k}$. We know that

$$
P_{A^{k}}=A^{k}\left(A^{k}\right)^{\dagger}=U\left[\begin{array}{cc}
I_{\rho} & 0 \\
0 & 0
\end{array}\right] U^{*}
$$

and also,

$$
X A^{k}=U\left[\begin{array}{ll}
X_{11} T^{k} & X_{11} \widetilde{T} \\
X_{21} T^{k} & X_{21} \widetilde{T}
\end{array}\right] U^{*} .
$$

Hence, the condition $P_{A^{k}} X A^{k}=X A^{k}$ implies $X_{21}=0$ because $T$ is nonsingular.

On the other hand, we write $Y$ as

$$
Y=U\left[\begin{array}{ll}
Y_{11} & Y_{12} \\
Y_{21} & Y_{22}
\end{array}\right] U^{*}
$$

according to the partition of $A$. Clearly, vectors of the form

$$
v=U\left[\begin{array}{l}
0 \\
y
\end{array}\right] \in \mathbb{C}^{n} \quad \text { with arbitrary } y \in \mathbb{C}^{n-\rho},
$$

belong to $\mathcal{N}\left(\left(A^{k}\right)^{*}\right)$. By (e), $v \in \mathcal{N}\left(A^{k} Y\right)$ holds. In consequence, from

$$
A^{k} Y=U\left[\begin{array}{cc}
T^{k} Y_{11}+\widetilde{T} Y_{21} & T^{k} Y_{12}+\widetilde{T} Y_{22} \\
0 & 0
\end{array}\right] U^{*},
$$

the condition $v \in \mathcal{N}\left(A^{k} Y\right)$ implies that $\left(T^{k} Y_{12}+\widetilde{T} Y_{22}\right) y=0$ is valid for arbitrary $y \in \mathbb{C}^{n-\rho}$. Thus, $T^{k} Y_{12}+\widetilde{T} Y_{22}=0$. It then follows that $Y_{12}=-\left(T^{k}\right)^{-1} \widetilde{T} Y_{22}$. Also, the condition $A^{k} Y A^{k}=A^{k}$ implies that $T^{k} Y_{11}+\widetilde{T} Y_{21}=I_{\rho}$, from where $Y_{11}=\left(T^{k}\right)^{-1}\left(I_{\rho}-\widetilde{T} Y_{21}\right)$.

Finally, the expressions for $X$ and $Y$ can be written as in item (d). This completes the proof.

Remark 3.3. Similarly to Remark 2.5, conditions (c) and (d) in Theorem 3.2 are equivalent to:

(c') $X=A^{d}+Z\left(I_{n}-P_{A^{k}}\right), \quad Y=\left(A^{k}\right)^{\dagger}+\left(I_{n}-Q_{A^{k}}\right) W$ for some $Z, W \in \mathbb{C}^{n \times n}$;

(d') $X$ and $Y$ can be expressed as in Theorem 3.2 (d) for some $X_{12} \in \mathbb{C}^{r \times(n-\rho)}, X_{22}, Y_{22} \in$ $\mathbb{C}^{(n-\rho) \times(n-\rho)}$, and $Y_{21} \in \mathbb{C}^{(n-\rho) \times \rho}$.

Since the Drazin inverse $X=A^{d}$ satisfies $X \in A\left\{5,6^{k}\right\}$ and the Moore-Penrose inverse $Y=\left(A^{k}\right)^{\dagger}$ belongs to $A^{k}\{1\}$, the above theorem contains (3.4) as a special case because the condition (b) holds. 
Theorem 3.4. Let $A \in \mathbb{C}^{n \times n}$ be a matrix of index $k$ written as in (3.1) with $\rho=\operatorname{rk}\left(A^{k}\right)$. Let $Z \in A^{k}\{1\}$. Then the following conditions are equivalent:

(a) $A^{\oplus}=\left(A^{k+1} Z\right)^{\dagger}$;

(b) $Z \in A^{k}\{3\}$;

(c) Z can be expressed as

$$
Z=U\left[\begin{array}{cc}
\left(T^{k}\right)^{-1}\left(I_{\rho}-\widetilde{T} Z_{21}\right) & -\left(T^{k}\right)^{-1} \widetilde{T} Z_{22} \\
Z_{21} & Z_{22}
\end{array}\right] U^{*}
$$

with $\widetilde{T}$ defined in (3.3) and for arbitrary $Z_{21} \in \mathbb{C}^{(n-\rho) \times \rho}$ and $Z_{22} \in \mathbb{C}^{(n-\rho) \times(n-\rho)}$.

(d) $A^{k} Z=P_{A^{k}}$.

(e) $Z=\left(A^{k}\right)^{\dagger}+\left(I_{n}-Q_{A^{k}}\right) W$, for arbitrary $W \in \mathbb{C}^{n \times n}$.

Proof. (b) $\Longleftrightarrow$ (c) It has been already proved in the proof of Theorem 3.2.

(b) $\Longleftrightarrow$ (d) It has been shown in Theorem 3.2.

(d) $\Longleftrightarrow$ (e) It follows from Lemma 2.1.

We show that (a) and (c) are equivalent. The condition $A^{\oplus}=\left(A^{k+1} Z\right)^{\dagger}$ is equivalent to

$$
\left(A^{\oplus}\right)^{\dagger}=A^{k+1} Z \text {. }
$$

We consider the following partition of $Z$

$$
Z=U\left[\begin{array}{ll}
Z_{11} & Z_{12} \\
Z_{21} & Z_{22}
\end{array}\right] U^{*}
$$

according to the size of blocks in $A$. Since $Z$ is an inner generalized inverse of $A^{k}, Z$ is of the form

$$
Z=U\left[\begin{array}{cc}
\left(T^{k}\right)^{-1}\left(I_{\rho}-\widetilde{T} Z_{21}\right) & Z_{12} \\
Z_{21} & Z_{22}
\end{array}\right] U^{*}
$$

and therefore

$$
A^{k+1} Z=U\left[\begin{array}{cc}
T^{k+1} & T \widetilde{T} \\
0 & 0
\end{array}\right]\left[\begin{array}{cc}
\left(T^{k}\right)^{-1}\left(I_{\rho}-\widetilde{T} Z_{21}\right) & Z_{12} \\
Z_{21} & Z_{22}
\end{array}\right] U^{*}=U\left[\begin{array}{cc}
T & T^{k+1} Z_{12}+T \widetilde{T} Z_{22} \\
0 & 0
\end{array}\right] U^{*}
$$

On the other hand, by using (3.2) we have

$$
\left(A^{\oplus}\right)^{\dagger}=U\left[\begin{array}{ll}
T & 0 \\
0 & 0
\end{array}\right] U^{*}
$$


Finally, from (3.8)-(3.10) follow that the equality $A^{\oplus}=\left(A^{k+1} Z\right)^{\dagger}$ holds if and only if $Z_{12}=-\left(T^{k}\right)^{-1} \widetilde{T} Z_{22}$.

We notice that the matrix $Z$ in (b) can be the Moore-Penrose inverse of $A^{k}$, and hence the above theorem contains the representation of the core EP inverse given in (1.3), that is, $A^{\oplus}=\left(A P_{A^{k}}\right)^{\dagger}$.

Remark 3.5. Similarly to Remark 2.5 , conditions (c) and (e) in Theorem 3.4 are equivalent to:

(c') $Z$ can be expressed as in Theorem 3.4 for some $Z_{21} \in \mathbb{C}^{(n-\rho) \times \rho}$ and $Z_{22} \in \mathbb{C}^{(n-\rho) \times(n-\rho)}$;

(e') $Z=\left(A^{k}\right)^{\dagger}+\left(I_{n}-Q_{A^{k}}\right) W$, for some $W \in \mathbb{C}^{n \times n}$.

\section{Maximal classes for matrices determining the CMP inverse}

In this section we study a representation for the CMP inverse obtained by Salemi and Mehdipour [9] and we derive maximal classes of matrices for which the representation remains valid.

Theorem 4.1. Let $A \in \mathbb{C}^{n \times n}$ be a matrix of index $k$ written as in (1.9). Let $r=\operatorname{rk}(A), X \in A\left\{5,6^{k}\right\}$, $Y, T \in A\{1\}$. The following conditions are equivalent:

(a) $A^{c, \dagger}=Y A X A T$;

(b) $A X A=A A^{d} A, Y A^{k}=A^{\dagger} A^{k}$, and $A^{k} T=A^{k} A^{\dagger}$;

(c) $X=A^{d}+Z-Q_{A} Z P_{A}, Y=A^{\dagger}+W\left(I_{n}-P_{A^{k}}\right)$, and $T=A^{\dagger}+\left(I_{n}-Q_{A^{k}}\right) V$, for arbitrary $Z, W, V \in \mathbb{C}^{n \times n}$;

(d) $X, Y$, and $T$ can be expressed as

$$
\begin{gathered}
X=U\left[\begin{array}{cc}
(\Sigma K)^{d}+\left(I_{r}-K^{*} K\right) X_{11}-K^{*} L X_{21} & X_{12} \\
-L^{*} K X_{11}+\left(I_{n-r}-L^{*} L\right) X_{21} & X_{22}
\end{array}\right] U^{*} \\
Y=U\left[\begin{array}{cc}
K^{*} \Sigma^{-1}+Y_{11}\left(I_{r}-(\Sigma K)^{k-1}\left((\Sigma K)^{k-1}\right)^{\dagger}\right) & Y_{12} \\
L^{*} \Sigma^{-1}+Y_{21}\left(I_{r}-(\Sigma K)^{k-1}\left((\Sigma K)^{k-1}\right)^{\dagger}\right) & Y_{22}
\end{array}\right] U^{*} \\
T=U\left[\begin{array}{cc}
K^{*} \Sigma^{-1}+\left(I_{r}-K^{*} M K\right) T_{11}-K^{*} M L T_{21} & \left(I_{r}-K^{*} M K\right) T_{12}-K^{*} M L T_{22} \\
L^{*} \Sigma^{-1}-L^{*} M K T_{11}+\left(I_{n-r}-L^{*} M L T_{21}\right. & M K T_{12}+\left(I_{n-r}-L^{*} M L\right) T_{22}
\end{array}\right] U^{*},
\end{gathered}
$$

with $M:=\left[(\Sigma K)^{k-1} \Sigma\right]^{\dagger}(\Sigma K)^{k-1} \Sigma$ and arbitrary $X_{11}, X_{12}, X_{21}, X_{22}, Y_{11}, Y_{12}, Y_{21}, Y_{22}, T_{11}, T_{12}$, $T_{21}, T_{22}$. 
Proof. (a) $\Longrightarrow$ (b) Since $A^{c, \dagger}=Q_{A} A^{d} P_{A}$ holds by definition, post- and premultiplying $A^{c, \dagger}=Y A X A T$ by $A$ we get $A A^{d} A=A X A$, because $Y, T \in A\{1\}$.

On the other hand, postmultiplying $A^{c, \dagger}=Y A X A T$ by $A^{k}$ and using that $P_{A} A^{k}=A^{k}$ we get

$$
Y A^{k}=A^{\dagger} A^{k}
$$

because $X \in A\left\{5,6^{k}\right\}$ and $T \in A\{1\}$. Similarly, premultiplying $A^{c, \dagger}=Y A X A T$ by $A^{k}$ and using $A^{k} Q_{A}=A^{k}, X \in A\left\{5,6^{k}\right\}$, and $Y \in A\{1\}$ we arrive at

$$
A^{k} T=A^{k} A^{\dagger} .
$$

(b) $\Longrightarrow$ (c) Applying Lemma 2.1 to the equation $A X A=A A^{d} A$ we get

$$
X=A^{d}+Z-Q_{A} Z P_{A}, \quad \text { for arbitrary } \quad Z \in \mathbb{C}^{n \times n},
$$

because $A^{d}$ is a particular solution.

Next, the proof of the part (c) follows directly from (4.4), (4.5), and Lemma 2.1.

(c) $\Longrightarrow$ (a) We know that $A^{\ell} Q_{A^{\ell}}=A^{\ell}$ and $P_{A^{\ell}} A^{\ell}=A^{\ell}$, for every positive integer $\ell$. So, particularizing for $\ell=1, \ell=k$, and taking into account that $A^{d} A=A^{k}\left(A^{d}\right)^{k}=\left(A^{d}\right)^{k} A^{k}$ we have

$$
\begin{aligned}
Y A X A T & =\left[A^{\dagger}+W\left(I_{n}-P_{A^{k}}\right)\right] A\left[A^{d}+Z-Q_{A} Z P_{A}\right] A\left[A^{\dagger}+\left(I_{n}-Q_{A^{k}}\right) V\right] \\
& =\left[A^{\dagger}+W\left(I_{n}-P_{A^{k}}\right)\right] A A^{d} A\left[A^{\dagger}+\left(I_{n}-Q_{A^{k}}\right) V\right] \\
& =Q_{A} A^{d} A\left[A^{\dagger}+\left(I_{n}-Q_{A^{k}}\right) V\right] \\
& =Q_{A} A^{d} P_{A}+Q_{A} A^{d} A\left(I_{n}-Q_{A^{k}}\right) V \\
& =A^{c, \dagger}
\end{aligned}
$$

(c) $\Longleftrightarrow$ (d) First, we write $Z$ by means of an block partition:

$$
Z=U\left[\begin{array}{ll}
Z_{11} & Z_{12} \\
Z_{21} & Z_{22}
\end{array}\right] U^{*}
$$

with appropriate sizes. From (2.2) we obtain

$$
P_{A}=U\left[\begin{array}{cc}
I_{r} & 0 \\
0 & 0
\end{array}\right] U^{*}, \quad Q_{A}=U\left[\begin{array}{cc}
K^{*} K & K^{*} L \\
L^{*} K & L^{*} L
\end{array}\right] U^{*} .
$$

In consequence, it is clear that $X=A^{d}+Z-Q_{A} Z P_{A}$ is valid if and only if (4.1) holds after renaming adequately some blocks.

On the other hand, as in (3.7) we have

$$
P_{A^{k}}=A^{k}\left(A^{k}\right)^{\dagger}=U\left[\begin{array}{cc}
(\Sigma K)^{k-1}\left((\Sigma K)^{k-1}\right)^{\dagger} & 0 \\
0 & 0
\end{array}\right] U^{*} .
$$


Now, partitioning adequately

$$
W=U\left[\begin{array}{ll}
W_{11} & W_{12} \\
W_{21} & W_{22}
\end{array}\right] U^{*},
$$

some direct calculations show that the expression $Y=A^{\dagger}+W\left(I_{n}-P_{A^{k}}\right)$ can be rewritten as in (4.2). Finally, as in the proof of Theorem 2.2 the expression $T=A^{\dagger}+\left(I_{n}-Q_{A^{k}}\right) V$ can be rewritten as in (4.3).

Remark 4.2. Similarly to Remark 2.5, conditions (c) and (d) in Theorem 4.1 are equivalent to:

(c') $X=A^{d}+Z-Q_{A} Z P_{A}, Y=A^{\dagger}+W\left(I_{n}-P_{A^{k}}\right)$, and $T=A^{\dagger}+\left(I_{n}-Q_{A^{k}}\right) V$, for some $Z, W, V \in \mathbb{C}^{n \times n}$

(d') $X, Y$, and $T$ can be expressed as in Theorem 4.1 for some $X_{11}, X_{12}, X_{21}, X_{22}, Y_{11}, Y_{12}, Y_{21}$, $Y_{22}, T_{11}, T_{12}, T_{21}, T_{22}$

Remark 4.3. (a) Since the Drazin inverse $A^{d}$ belongs to $A\left\{5,6^{k}\right\}$, and the Moore-Penrose inverse $A^{\dagger}$ belongs to $A\{1\}$, the above theorem recovers (1.6) as a particular case.

(b) Under the assumptions of Theorem 4.1, we notice that if $k=1$ then $X \in A\{1\}$. So, [9, Theorem 2.4] implies that $A^{\dagger}=A^{c, \dagger}=Y A X A T=Y A T$ with $Y, T \in A\{1\}$. On the other hand, if $Z \in\left(A A^{*}\right)\{1\}$ and $W \in\left(A^{*} A\right)\{1\}$, it is well known that $A^{*} Z \in A\{1\}$ and $W A^{*} \in A\{1\}$ by applying the rank cancellation rule [12, p. 145]. In consequence, setting $Y=A^{*} Z$ and $T=W A^{*}$ we obtain $A^{\dagger}=A^{*} Z A W A^{*}$, which is a representation for the Moore Penrose inverse known as the Decell's formula as indicated in (1.8). Further, also we derive the Urquhart's formula, that is $A^{\dagger}=A^{(1,4)} A A^{(1,3)}$ as indicated in (1.7). In fact, it is easy to see that $A^{*} Z \in A\{1,4\}$ and $W A^{*} \in A\{1,3\}$, again by the rank cancellation rule.

\section{Acknowledgements}

We thank the referees for carefully reading our paper and for their comments and suggestions that have made the paper better.

\section{References}

[1] A. Ben-Israel, T.N.E Greville, Generalized Inverses: Theory and Applications, second ed., Springer-Verlag, New York, 2003. 
[2] O.M. Baksalary, G. Trenkler, Core inverse of matrices, Linear and Multilinear Algebra, 58 (6) (2010) 681-697.

[3] H.P. Decell, An alternate form of the generalized inverse of an arbitrary complex matrix, SIAM Review, 7 (3) (1965) 356-358.

[4] D.E. Ferreyra, F.E. Levis, N. Thome, Revisiting of the core EP inverse and its extension to rectangular matrices, Quaestiones Mathematicae, 41 (2) (2018) 265-281.

[5] C.H. Hung, T.L. Markham, The Moore-Penrose inverse of a partitioned matrix $M=\left[\begin{array}{ll}A & B \\ C & D\end{array}\right]$, Linear Algebra and its Applications, 11 (1) (1975) 73-86.

[6] H. Kurata, Some theorems on the core inverse of matrices and the core partial ordering, Applied Mathematics and Computation, 316 (2018) 43-51.

[7] S.B. Malik, Some more properties of core partial order, Applied Mathematics and Computation, 221 (2013) 192-201.

[8] S. Malik, N. Thome, On a new generalized inverse for matrices of an arbitrary index, Applied Mathematics and Computation, 226 (2014) 575-580.

[9] M. Mehdipour, A. Salemi, On a new generalized inverse of matrices, Linear and Multilinear Algebra, 66 (5) (2018) 1046-1053.

[10] S.K. Mitra, On a generalized inverse of matrix and applications, Sankhyā: The Indian Journal of Statistics, Series A, 30 (1) (1968) 107-114.

[11] K.M. Prasad, K.S. Mohana, Core EP inverse, Linear and Multilinear Algebra, 62 (3) (2014) $792-802$.

[12] S. Puntanen, G.P.H. Styan, J. Isotalo, Matrix tricks for linear statistical models, Springer, New York, 2011.

[13] X. Wang, Core-EP decomposition and its applications, Linear Algebra and its Applications, 508 (2016) 289-300.

[14] D. Mosić, Core-EP pre-order of Hilbert space operators, Quaestiones Mathematicae, doi: 10.2989/16073606.2017.1393021 (2017). 
[15] S.Z. Xu, J.L. Chen, X.X. Zhang. New characterizations for core inverses in rings with involution, Frontiers of Mathematics in China, 12 (2017) 231-246.

[16] S. Zlovec, An explicit form of the Moore-Penrose inverse of an arbitrary complex matrix, SIAM Review, 12 (1970) 132-134. 